\title{
Remarks on Existence/Nonexistence of Analytic Solutions to Higher Order KdV Equations
}

\author{
A. KARCZEWSKA ${ }^{a, *}$ AND P. ROZMEJ ${ }^{b}$ \\ ${ }^{a}$ Faculty of Mathematics, Computer Science and Econometrics University of Zielona Góra, \\ Z. Szafrana 4a, 65-246 Zielona Góra, Poland \\ ${ }^{b}$ Institute of Physics, Faculty of Physics and Astronomy University of Zielona Góra, \\ Z. Szafrana 4a, 65-246 Zielona Góra, Poland
}

(Received June 5, 2019; in final form October 15, 2019)

This paper is dedicated to the memory of our friend Professor Eryk Infeld (1940-2019), who recently passed away.

\begin{abstract}
In this note, we discuss the existence of analytic solutions to the nonlinear wave equations of the higher order than the ubiquitous Korteweg-de Vries equation. First, we recall our recent results which show that the extended Korteweg-de Vries equation, that is, the equation obtained within second-order perturbation approach possesses three kinds of analytic solutions. These solutions have the same functional form as the corresponding Korteweg-de Vries solutions. We show, however, that the most intriguing multi-soliton solutions, known for the Korteweg-de Vries equation, do not exist for extended Korteweg-de Vries equation. Moreover, we show that for the equations obtained in the third order perturbation approach (and then in any higher order) analytic solutions in the forms known from Korteweg-de Vries theory do not exist.
\end{abstract}

DOI: 10.12693/APhysPolA.136.910

PACS/topics: shallow water waves, extended KdV equations, analytic solutions

\section{Introduction}

The general problem of fluid motion with arbitrary boundary conditions leads to a set of the Navier-Stokes equations. In most cases attempts to solve these equations lead to extremely difficult problems. Therefore in many cases, some simplified models are introduced. For shallow water problem, physicists use the ideal fluid model. This means that fluid is assumed to be incompressible and inviscid with additional assumption that the fluid motion is irrotational. Since in normal conditions water viscosity and compressibility are very small, the model should reproduce the fluid motion with reasonable accuracy, until waves on the surface do not break. Therefore, for ideal fluid one obtains a system of four partial differential equations on two unknown functions, the velocity potential $\phi(x, y, z, t)$ and the surface elevation $\eta(x, y, t)$. This system contains the Laplace equation for velocity potential, kinematic and dynamic boundary conditions at the (unknown) surface, and the kinematic boundary condition at the bottom. At this stage coordinates and time are dimensional quantities.

The introduction of scaled dimensionless coordinates to the system of the hydrodynamic Euler equations allows us to apply a perturbation approach. The solution of the velocity potential is assumed in the form of power series with respect to the vertical coordinate, which when the Laplace equation is applied results in power series in

\footnotetext{
* corresponding author; e-mail A.Karczewska@wmie.uz.zgora.pl
}

the small parameter $\beta=\left(\frac{h}{l}\right)^{2}$. Here, $h$ denotes the water depth, $l$ is an average wavelength. The boundary conditions at the hard bottom and the free surface introduce dependence on the second small parameter $\alpha=\frac{A}{h}$, where $A$ is the amplitude of the surface wave. The final wave equation for the surface wave can be obtained in different orders of the perturbation approach. It is worth noticing that the result of the perturbation approach depends on the relation between the small parameters. The case when $\alpha=O(\beta)$, that is, $\alpha$ and $\beta$ are of the same order, corresponds to weakly dispersive nonlinear waves and leads to the KdV equation [1]. It is worth noticing that the KdV equation appears to be first order nonlinear wave equation in several different systems, like multilayer fluids, ion acoustic waves in plasma, electric circuits, and propagation of optical impulses in fibers, among others, see, e.g. monographs $[2-4]$.

In the fixed reference frame and scaled dimensionless coordinates the $\mathrm{KdV}$ equation has the following form (indexes denote partial derivatives, e.g., $\left.\eta_{3 x} \equiv \frac{\partial^{3} \eta}{\partial x^{3}}\right)$ :

$$
\eta_{t}+\eta_{x}+\frac{3}{2} \alpha \eta \eta_{x}+\frac{1}{6} \beta \eta_{3 x}=0 .
$$

It is well known that the $\mathrm{KdV}$ equation is integrable and possesses an infinite number of invariants.

When the perturbation approach is continued to second order, the resulted equation is known as the extended $K d V[5]$ or $K d V$ 2:

$$
\begin{aligned}
& \eta_{t}+\eta_{x}+\alpha \frac{3}{2} \eta \eta_{x}+\beta \frac{1}{6} \eta_{3 x}-\alpha^{2} \frac{3}{8} \eta^{2} \eta_{x} \\
& +\alpha \beta\left(\frac{23}{24} \eta_{x} \eta_{2 x}+\frac{5}{12} \eta \eta_{3 x}\right)+\beta^{2} \frac{19}{360} \eta_{5 x}=0 .
\end{aligned}
$$


The KdV2 equation is not integrable. It has only one exact invariant (mass). There exist, however, a number of adiabatic invariants, which are constant up to second order in small parameters, see. e.g. [6, 7].

Taking into account all terms up to the third order in small parameters one arrives at the following $K d V 3$ equation

$$
\begin{aligned}
\eta_{t} & +\eta_{x}+\alpha \frac{3}{2} \eta \eta_{x}+\beta \frac{1}{6} \eta_{3 x}-\alpha^{2} \frac{3}{8} \eta^{2} \eta_{x} \\
& +\alpha \beta\left(\frac{23}{24} \eta_{x} \eta_{2 x}+\frac{5}{12} \eta \eta_{3 x}\right)+\beta^{2} \frac{19}{360} \eta_{5 x} \\
& +\alpha^{3} \frac{3}{16} \eta^{3} \eta_{x}+\alpha^{2} \beta\left(\frac{19}{32} \eta_{x}^{3}+\frac{23}{16} \eta \eta_{x} \eta_{2 x}+\frac{5}{16} \eta^{2} \eta_{3 x}\right) \\
& +\alpha \beta^{2}\left(\frac{317}{288} \eta_{2 x} \eta_{3 x}+\frac{1079}{1440} \eta_{x} \eta_{4 x}+\frac{19}{80} \eta \eta_{5 x}\right) \\
& +\beta^{3} \frac{55}{3024} \eta_{7 x}=0 .
\end{aligned}
$$

Equations (1)-(3) are valid in the fixed reference frame. Mathematicians prefer simpler versions of $\mathrm{KdV}$ obtained by a transformation to a frame moving with a natural velocity (equal to 1 in dimensionless coordinates or $\sqrt{g h}$ in dimension coordinates). For instance, in the case of $\beta=\alpha$, the following change of variables:

$$
\hat{x}=\sqrt{\frac{3}{2}}(x-t), \quad \hat{t}=\frac{1}{4} \sqrt{\frac{3}{2}} \alpha t \quad \text { and } \quad u=\eta
$$

transforms (1) into

$$
u_{\hat{t}}+6 u u_{\hat{x}}+u_{3 \hat{x}}=0,
$$

commonly used in mathematical papers.

The paper is organized as follows. In Sect. 2 we give a short overview of analytic solutions to $\mathrm{KdV}$. In Sect. 3 the analytic solutions to KdV2, which have the same functional forms as the corresponding $\mathrm{KdV}$ solutions, are presented. We show that for KdV2 exact multi-soliton solutions do not exist. In Sect. 4 we show that there are no analytic solutions for any higher order $\mathrm{KdV}$ equations.

\section{KdV and its solutions}

The KdV equation possesses many miraculous properties. The most striking is the existence of an infinite number of integral invariants which correspond to conservation laws. There exist several kinds of analytic solutions to $\mathrm{KdV}$ : single soliton solutions, periodic (cnoidal) solutions, periodic "superposition" solutions, and multisoliton solutions. Two first kinds of these solutions can be obtained by direct integration (see, e.g., monographs $[2,3])$. Below we show another method of obtaining single soliton and periodic solutions, applicable not only to KdV but also to KdV2. This method allows us to compare solutions of the same kind for equations of a different order.

\subsection{Single soliton solutions}

Assume solutions of the $\mathrm{KdV}$ equation in the form

$$
\eta(x, t)=A \operatorname{sech}^{2}[B(x-v t)]=A \operatorname{sech}^{2}(B y),
$$

where $y=x-v t$. Substitution of (4) into KdV (see Eq. (1)) gives

$$
-\frac{1}{3} A B \tanh (B y) \operatorname{sech}^{4}(B y)\left[G_{0}+G_{2} \cosh (2 B y)\right]=0 .
$$

Equation (5) is valid for any argument only when simultaneously

$$
G_{0}=3-3 v+9 \alpha A-10 \beta B^{2}=0,
$$

and

$$
G_{2}=3-3 v+2 \beta B^{2}=0 .
$$

This gives immediately

$$
B=\sqrt{\frac{3 \alpha}{4 \beta} A}, \quad v=1+\frac{\alpha}{2} A
$$

and the solution coincides with that obtained by direct integration of (1).

Remark 1 It is clear from (8) that solutions exist for arbitrary parameters $\alpha, \beta$. Since KdV imposes only two constraints on three coefficients $A, B, v$, there exists a one-parameter family of solutions. Usually, the amplitude $A$ can be considered arbitrary, within the range in which $\alpha$ stays small.

\subsection{Periodic (cnoidal) solutions}

In this case, the solution can be postulated in the form of the cnoidal wave

$$
\eta(x, t)=A \mathrm{cn}^{2}(B(x-v t), m)+D=A \mathrm{cn}^{2}(B y, m)+D .
$$

The constant $D$ is necessary in order to assure that the mean surface elevation coincides with undisturbed water level.

Equivalently, instead of the Jacobi elliptic cn function, dn or sn Jacobi elliptic functions can be used. Then, substitution of (9) into $\mathrm{KdV}$ yields equation analogous to (5):

$$
\frac{1}{3} A B \text { cn sn dn }\left(G_{0}+G_{2} \mathrm{cn}^{2}\right)=0 .
$$

In $(10)$, the arguments $(B(x-v t), m)$ of the Jacobi elliptic functions are omitted for abbreviation. So, there must be

$$
G_{0}=4 \beta B^{2}-8 \beta B^{2} m-9 \alpha D+6 v-6=0,
$$
and

$$
G_{2}=12 \beta B^{2} m-9 \alpha A=0 .
$$

Equation $G_{2}=0$ implies

$$
B=\sqrt{\frac{3 \alpha}{4 \beta} \frac{A}{m}} .
$$

Volume conservation condition determines

$$
D=-\frac{A}{m}\left[\frac{E(m)}{K(m)}+m-1\right] \text {. }
$$


In (14), $E(m)$ and $K(m)$ are the complete elliptic integral and the complete elliptic integral of the first kind, respectively. Then from $G_{0}=0$ one has

$$
v=1+\frac{\alpha A}{2 m}\left[2-m-3 \frac{E(m)}{K(m)}\right] \text {. }
$$

Remark 2 In the case of cnoidal solutions, $K d V$ with volume conservation condition supply three constraints on five parameters $A, B, v, D, m$. Then there is some freedom in allowable ranges of the coefficients. Usually, the amplitude $A$ is considered arbitrary, until there is no contradiction with the condition that $\alpha$ is small. In principle, the elliptic parameter can take values from the whole interval $m \in[0,1]$.

\subsection{Periodic "superposition" solutions}

Assume solutions to $\mathrm{KdV}$ in the form

$\eta_{ \pm}(y)=\frac{A}{2}\left[\operatorname{dn}^{2}(B y, m) \pm \sqrt{m} \operatorname{cn}(B y, m) \operatorname{dn}(B y, m)\right]+D$.

Coefficient $D$ is necessary in order to maintain, for arbitrary $m$, the same volume for a wave's elevations and depressions with respect to the undisturbed water level. This form of the solution (without $D$ term) has been proposed only recently in [8].

Insertion of (16) into (1) gives equation analogous to (5), which after some simplifications takes the form $F_{0}+F_{2} \mathrm{cn}^{2}+F_{11} \mathrm{cndn}=0$. Then there are three conditions on the solution

$$
\begin{aligned}
& F_{0}=9 \alpha A(1-m)+2 \beta B^{2}(5 m-1) \\
& \quad+18 \alpha D+12(1-v)=0, \\
& F_{2}=9 \alpha A m-12 \beta B^{2} m=0, \\
& F_{11}=9 \alpha A \sqrt{m}-12 \beta B^{2} \sqrt{m}=0 .
\end{aligned}
$$

Equations (18) and (19) are equivalent and yield the same

$$
B=\sqrt{\frac{3 \alpha}{4 \beta} A} .
$$

Then volume conservation condition together with (17) determine $D$ and $v$ as functions of $A$ and $m$ :

$D=-\frac{A}{2} \frac{E(m)}{K(m)} \quad$ and $\quad v=1+\frac{\alpha A}{8}\left[5-m-6 \frac{E(m)}{K(m)}\right]$.

The solutions $\eta_{ \pm}(16)$ are different than those given by (9). However, Remark 2 applies to these solutions, as well.

\subsection{Multi-soliton solutions}

The existence of multi-soliton solutions is one of the most exciting properties of the KdV equation. Zabusky and Kruskal [9] noticed the first indication of that property in their famous numerical experiment. The paper [9] inspired intensive studies which resulted in the development of a general method, by Gardner et al. [10], called inverse scattering transform (IST). The IST allows us to construct the whole family of multi-soliton solutions. There exist also other methods for construction of multisoliton solutions (e.g., the Bäcklund transformations, the Lax pairs, the Hirota direct method). During their motion solitons having different velocities collide and regain their shapes after reseparation. KdV equation permits for the existence of solitons with different amplitudes (and therefore different velocities). Therefore analytic multi-soliton solutions can exist.

\section{Analytic solutions to $\mathrm{KdV2}$}

In this section, we discuss the solutions to KdV2 Eq. (2) derived by us in the same way as solutions to $\mathrm{KdV}$ (1) in Sect. 2. In Refs. [11-15] we have shown that for the KdV2 equation there exist analytic solutions of the same forms as solutions to $\mathrm{KdV}(4),(9)$ and (16) but with different coefficients $A, B, D, v$. Here, we give a brief overview of these results, the full presentation of which is contained in [11-15].

\subsection{Single soliton solution to KdV2}

Substitution of the postulated form of the solution (4) into (2) leads (after some simplifications) to the equation analogous to (5) but containing $C_{0}+C_{2} \operatorname{sech}^{2}(B y)+$ $C_{4} \operatorname{sech}^{4}(B y)$ in the square bracket. This implies three linearly independent equations for three unknowns coefficients $A, B, v$ :

$$
\begin{aligned}
& (1-v)+\frac{2}{3} B^{2} \beta+\frac{38}{45} B^{4} \beta^{2}=0, \\
& \frac{3 A \alpha}{4}-B^{2} \beta+\frac{11}{4} A \alpha B^{2} \beta-\frac{19}{3} B^{4} \beta^{2}=0, \\
& -\left(\frac{1}{8}\right)(A \alpha)^{2}-\frac{43}{12} A \alpha B^{2} \beta+\frac{19}{3} B^{4} \beta^{2}=0 .
\end{aligned}
$$

From (24), denoting $z=\frac{\beta B^{2}}{\alpha A}$ one has

$$
\frac{19}{3} z^{2}-\frac{43}{12} z-\frac{1}{8}=0,
$$

with roots

$$
z_{1}=\frac{43-\sqrt{2305}}{152} \approx-0.033<0
$$

and

$$
z_{2}=\frac{43+\sqrt{2305}}{152} \approx 0.599>0 .
$$

Since for soliton solution $A>0$, only $z=z_{2}$ has physical relevance $\left(B^{2}>0\right)$. In this case (see $\left.[11,15]\right)$, all three coefficients of the solution are fixed by the coefficients of the KdV2 equation $\alpha, \beta$ :

$$
\begin{aligned}
& A \approx \frac{0.242399}{\alpha}>0, \quad B \approx \sqrt{\frac{0.145137}{\beta}}, \\
& v \approx 1.11455 .
\end{aligned}
$$

The result (28) means that contrary to the KdV case, for KdV2 exists the unique soliton solution (4) with coefficients $A, B, v$ determined by the equation parameters $(\alpha, \beta)$. 


\subsection{Periodic cnoidal solutions to KdV2}

Substitution of the postulated form of the solution (9) into (2) leads, similarly as in the soliton case discussed in Sect. 3.1, to three linearly independent equations for four unknowns coefficients $A, B, D, v$ which have to be supplemented by volume conservation condition. The last one gives the relation $D=-\frac{A}{m}\left[\frac{E(m)}{K(m)}+m-1\right]$, analogous to that in (21). Substitution $z=\frac{B^{2} \beta}{A \alpha} m$ transforms one of the equations into (25). In this case, however, both positive and negative $z$ roots $(26)$ can be relevant. For both $z$-roots the explicit formulae for $A, B, D, v$ are obtained as functions of $\alpha, \beta$ and the elliptic parameter $m$. Although from mathematical viewpoint all values of $m \in[0,1]$ are admissible, the definition of small parameter $\alpha$ requires that the amplitude coefficient $A$ cannot be much greater than one (in scaled coordinates). This requirement puts limits on the physically relevant values of the elliptic parameter $m$.

For the case $z=z_{2}$, physically reasonable values of $A$ occur only in a narrow interval of $m$ close to 1 (remember that when $m \rightarrow 1$ the space period of cnoidal wave tends to infinity and the solution tends to the soliton solution). These solutions are "normal" cnoidal waves with the crests up and the troughs down, slightly different from cnoidal $\mathrm{KdV}$ solutions.

For the case $z=z_{1}$, however, the cnoidal $\mathrm{KdV} 2$ solutions exhibit a new feature. $B$ is real for negative $A$. This fact means that the solutions are inverted cnoidal waves, with the crests down and the troughs up. The reasonable values of $|A|$ occur only for small $m \in[0, \approx 0.2)$. Therefore shapes of such waves are not much different from the usual cosine waves. For more details, we refer to $[12,15]$.

\subsection{Periodic "superposition" solutions to KdV2}

In this case, the general procedure for obtaining the solutions of $\mathrm{KdV} 2$ equation is analogous to that for the $\mathrm{KdV}$ case, described in Sect. 2.3. Insertion of $\eta_{ \pm}$into the KdV2 Eq. (2) supplies now the equation of the form (arguments $(B y, m)$ omitted)

$$
F_{0}+F_{2} \mathrm{cn}^{2}+F_{4} \mathrm{cn}^{4}+F_{11} \mathrm{cn} \mathrm{dn}+F_{31} \mathrm{cn}^{3} \mathrm{dn}=0 .
$$

It appears, however, that only three of equations $F_{i}=0$ are linearly independent and with volume conservation condition constitute four equations for unknown coefficients of solutions. Substitution $z=\frac{B^{2} \beta}{A \alpha}$ transforms one of these equations into (25). Then, similarly as in the case of cnoidal solutions, two cases occur, when $z=z_{1}$ and $z=z_{2}$. Finally, the "superposition" solutions to KdV2 are qualitatively similar to cnoidal solutions, but with slightly different amplitudes, velocities and wavelengths. For more details, we refer to [13-15].

\subsection{Nonexistence of multi-soliton solutions to KdV2}

The given KdV2 equation (given values of $\alpha, \beta$ parameters) determines the unique single soliton solution (presented in Sect. 3.1). This fact means that for KdV2 there is no room for solitons moving with different velocities (and amplitudes). Therefore, multi-soliton solutions to KdV2 cannot exist.

\section{Nonexistence of analytic solutions to $\mathrm{KdV3}$}

Searching for single soliton solutions we substitute the postulated form of the solution (4) into KdV3 (3). The result can be transformed into the equation

$$
\begin{aligned}
& C_{0}+C_{2} \cosh ^{2}(B(x-v t))+C_{4} \cosh ^{4}(B(x-v t)) \\
& \quad+C_{6} \cosh ^{6}(B(x-v t))=0,
\end{aligned}
$$

where all $C_{i}$ are functions of $A, B, v$. Equation (30) implies four conditions $C_{0}=C_{2}=C_{4}=C_{6}=0$. The explicit form of these conditions is the following:

$$
\begin{aligned}
& C_{0}=9 \alpha^{3} A^{3}-708 \alpha^{2} A^{2} \beta B^{2}+12224 \alpha A \beta^{2} B^{4} \\
& \quad-17600 \beta^{3} B^{6}=0 \\
& C_{2}=9 \alpha^{2} A^{2}\left(25 \beta B^{2}-1\right)-2 \alpha A \beta B^{2}\left(2714 \beta B^{2}+129\right) \\
& \quad+8 \beta^{2} B^{4}\left(1100 \beta B^{2}+57\right)=0 \\
& C_{4}=3 \alpha A\left(334 \beta^{2} B^{4}+55 \beta B^{2}+15\right) \\
& \quad-20 \beta B^{2}\left(110 \beta^{2} B^{4}+19 \beta B^{2}+3\right)=0 \\
& C_{6}=1100 \beta^{3} B^{6}+798 \beta^{2} B^{4}+630 \beta B^{2} \\
& \quad-945 v+945=0 .
\end{aligned}
$$

Equations (31)-(33) are linearly independent with two unknowns $A, B$. Since the number of equations exceeds the number of unknowns, they appear to be contradictory. Therefore, single soliton solutions of the form (4) to KdV3 do not exist.

Proceeding in the analogous way with search for cnoidal solutions (9) one reaches the similar structure of four conditions $C_{0}=C_{2}=C_{4}=C_{6}=0$. However, volume conservation condition supplies the fifth equation. In this case, there are four unknowns $A, B, v$ and $D$. Again, similarly as in the previous case, the five equations for the unknown coefficients appear to be contradictory.

Qualitatively the same contradiction of equations is obtained for coefficients of "superposition" solutions to KdV3. In this case, the number of linerly independent equations exceeds the number of unknowns, as well.

Conclusion: For the KdV3 equation single soliton solutions of the form (1), cnoidal solutions of the form (9) and "superposition" solutions of the form (16) do not exist.

\section{The fifth order $\mathrm{KdV}$ equation}

The fifth order $\mathrm{KdV}$ equation was derived in [16] for capilary-gravity surface waves. In scaled dimensionless coordinates coordinates it has the following form:

$$
\eta_{t}+\eta_{x}+\frac{3}{2} \alpha \eta \eta_{x}+\beta \tau_{1} \eta_{3 x}+\beta^{2} \tau_{2} \eta_{5 x}=0,
$$


where

$$
\tau_{1}=\frac{1-3 \tau}{6} \quad \text { and } \quad \tau_{2}=\frac{19-30 \tau-45 \tau^{2}}{360} .
$$

In Eq. (36), $\tau=\frac{T}{\varrho g h^{2}}$ is the bond number, and $T$ is the surface tension coefficient. It is known, see, e.g., [17-19], that when $\tau>\frac{1}{3}$ Eq. (35) has the soliton solution of the form

$$
\eta(x, t)=A \operatorname{sech}^{4}[B(x-v t)] .
$$

It is worth to show an example of derivation of the solution (37). Equation (35), after variable change $y=$ $x-v t$, becomes ODE. It can be integrated to the form

$$
(1-v) \eta+\frac{3}{4} \eta \eta_{y}+\beta \tau_{1} \eta_{2 y}+\beta^{2} \tau_{2} \eta_{4 y}=0 .
$$

The constant on the rhs has to be zero since

$$
\lim _{y \rightarrow \pm \infty} \eta=0 \text {. }
$$

Insertion of the postulated solution (37) into (38) yields

$$
C_{0}+C_{2} \operatorname{sech}^{2}(B y)+\operatorname{sech}^{4}(B y)=0,
$$

which supplies three conditions $C_{i}=0$ to be satisfied simultaneously. So, we have

$$
\begin{aligned}
& C_{0}=4-4 v+64 B^{2} \tau_{1} \beta+1024 B^{4} \tau_{2} \beta^{2}=0, \\
& C_{2}=-80 B^{2} \tau_{1} \beta-4160 B^{4} \tau_{2} \beta^{2}=0, \\
& C_{4}=3 A \alpha+3360 B^{4} \tau_{2} \beta^{2}=0 .
\end{aligned}
$$

Solving the set $(40)-(42)$ one obtains

$$
\begin{aligned}
& A=-\frac{700(1-3 \tau)^{2}}{169 \alpha\left(19-30 \tau-45 \tau^{2}\right)}, \\
& B=\sqrt{-\frac{15(1-3 \tau)}{13 \beta\left(19-30 \tau-45 \tau^{2}\right)},} \\
& v=\frac{2851-2910 \tau-10845 \tau^{2}}{169\left(19-30 \tau-45 \tau^{2}\right)} .
\end{aligned}
$$

The coefficient $B$ is real when $\tau \in\left[\frac{1}{3}, \frac{\sqrt{120}-5}{15} \approx 0.397\right]$.

Let us show an example of this solution for $\alpha=0.2$, $\beta=0.15$ and $\tau=0.36$. Come back to dimension variables for water wave case. For water $T \approx 72 \frac{m N}{m}$. So, in this case $h=\sqrt{\frac{T}{\varrho g \tau}} \approx 0.0045 \mathrm{~m}$. The soliton amplitude is $\alpha h \approx 0.0009 \mathrm{~m}$ and $x$ unit corresponds to $0.0117 \mathrm{~m}$. Indeed, it is capillary-gravity soliton on the surface of a thin water layer.

Figure 1 displays the motion of single soliton solution (37) according to the fifth order KdV Eq. (35).

In the case of KdV2 equation besides single soliton solution of the form $\sim \operatorname{sech}^{2}[B(x-v t)]$ there exist periodic cnoidal solutions $\sim \mathrm{cn}^{2}[B(x-v t), m$. In analogy to the case of the KdV2 we can search for cnoidal periodic solution in the form

$\eta(x, t)=A \mathrm{cn}^{4}[B(x-v t), m]+D=A \mathrm{cn}^{4}[B y, m]+D$.

Insertion of (46) into (35) supplies equation analogous to $(30)$ :

$C_{0}+C_{2} \mathrm{cn}^{2}[B y, m]+C_{4} \mathrm{cn}^{4}[B y, m]+C_{6} \mathrm{cn}^{6}[B y, m]=0$.

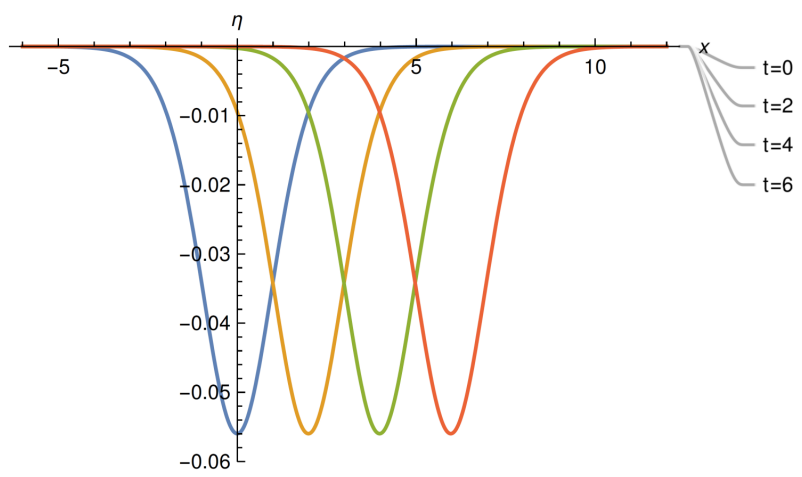

Fig. 1. Profiles of the soliton (37) for $t=0,3,4,6$ when $\alpha=0.2, \beta=0.15$ and $\tau=0.36$.

In order for (46) to be the solution of Eq. (35) all $C_{i}$ coefficients must be zero

$$
\begin{aligned}
& C_{0}=24 \beta B^{2}\left(-20 \beta B^{2} \tau_{2}\left(2 m^{2}-3 m+1\right)\right. \\
& \left.\quad+\tau_{1}(1-m)\right)=0, \\
& C_{2}=\beta^{2} B^{4} \tau_{2}\left(6784 m^{2}-6784 m+1024\right) \\
& \quad+\beta B^{2} \tau_{1}(128 m-64) 3 \alpha D-4 v+4=0, \\
& C_{4}=-120 \beta B^{2} m\left(52 \beta B^{2}(2 m-1) \tau_{2}+\tau_{1}\right), \\
& C_{6}=3 \alpha A+6720 \beta^{2} B^{4} m^{2} \tau_{2} .
\end{aligned}
$$

From (48) nonzero root of $B^{2}$ is

$B^{2}=\frac{\tau_{1}}{20 \beta \tau_{2}(1-2 m)}=3 \frac{(1-3 \tau)}{\beta(1-2 m)\left(19-30 \tau-45 \tau^{2}\right)}$,

whereas from (50) one has nonzero root

$B^{2}=\frac{\tau_{1}}{52 \beta \tau_{2}(1-2 m)}=\frac{15}{13} \frac{(1-3 \tau)}{\beta(1-2 m)\left(19-30 \tau-45 \tau^{2}\right)}$.

This is an obvious contradiction. Therefore Eqs. (48)(51) cannot be satisfied simultaneously. So, the fifth order KdV Eq. (35) does not possess cnoidal solution in the form (46).

Assuming periodic solution in the form

$$
\eta(x, t)=A \operatorname{cn}^{2}[B(x-v t)]+D,
$$

and proceeding as above we obtain that conditions analogous to (48)-(51) are contradictory, as well. Therefore, the function of the form (54) cannot be a solution to Eq. (35).

\section{Conclusions}

The main results of these studies can be summarized as follows.

- The extended KdV (KdV2) equation does not possess multi-soliton solutions. It possesses three kinds of analytic solutions of the same functional 
forms as $\mathrm{KdV}$ solutions, namely single soliton, cnoidal, and 'superposition solutions. However, multi-soliton solutions for KdV2 do not exist.

- For KdV3 Eq. (3), analytic solutions of the same functional forms as $\mathrm{KdV}$, and $\mathrm{KdV} 2$ solutions do not exist.

- The fifth order KdV Eq. (35) has an analytic solution in the form of a hyperbolic $\operatorname{sech}^{4}$ function. However, periodic solutions of (35) in the form of the Jacobi elliptic function $\mathrm{cn}^{2}$ or $\mathrm{cn}^{4}$ do not exist.

\section{References}

[1] D.J. Korteweg, G. de Vries, Philos. Mag. 39, 422 (1985).

[2] G.B. Whitham, Linear and Nonlinear Waves, Wiley, 1974.

[3] M. Dingemans, Water Wave Propagation over Uneven Bottoms, World Sci., Singapore 1997.

[4] E. Infeld, G. Rowlands, Nonlinear Waves, Solitons and Chaos, Cambridge University Press, 1990.

[5] T.R. Marchant, N.F. Smyth, J. Fluid Mech. 221, 263 (1990).

[6] A. Karczewska, P. Rozmej, E. Infeld, G. Rowlands, Phys. Lett. A 381, 270 (2017).

[7] P. Rozmej, A. Karczewska, in: Nonlinear Systems, Vol. 1, Mathematical Theory and Computational Methods, Eds. V. Carmona, J. Cuevas-Maraver, F. Fernández-Sánchez, E. Garcı-Medina, Springer, 2018, p. 175.
[8] A. Khare, A. Saxena, Phys. Lett. A 377, 2761 (2013).

[9] N.J. Zabusky, M.D. Kruskal, Phys. Rev. Lett. 15, 240 (1965).

[10] C.S. Gardner, J.M. Greene, M.D. Kruskal, R.M. Miura, Phys. Rev. Lett. 19, 1095 (1967).

[11] A. Karczewska, P. Rozmej, E. Infeld, Phys. Rev. E 90, 012907 (2014).

[12] E. Infeld, A. Karczewska, G. Rowlands, P. Rozmej, Acta Phys. Pol. A 133, 1191 (2018).

[13] P. Rozmej, A. Karczewska, E. Infeld, Nonlin. Dynam. 91, 1085 (2018)

[14] P. Rozmej, A. Karczewska, Adv. Math. Phys. 2018, 5095482 (2018).

[15] A. Karczewska, P. Rozmej, Shallow Water Waves - Extended Korteweg-de Vries Equations, Oficyna Wydawnicza Uniwersyteu Zielonogórskiego, Zielona Góra 2018.

[16] J.K. Hunter, J. Scheurle, Physica D 32, 253 (1988).

[17] R. Grimshaw, B. Malomed, E. Benilov, Physica D 77, 473 (1994).

[18] B. Dey, A. Khare, C.N. Kumar, Phys. Lett. A 223, 449 (1996).

[19] T.J. Bridges, G. Derks, G. Gottwald, Physica D 172 , $190(2002)$. 